\title{
Strong coupling expansion for Yang-Mills theory at finite temperature
}

\author{
Jens Langelage*, Gernot Münster, Owe Philipsen \\ Westfälische Wilhelms-Universität Münster, Institut für Theoretische Physik, \\ Wilhelm-Klemm-Str. 9, 48149 Münster, Germany \\ E-mail: jens.langelage@uni-muenster.de \\ E-mail: munsteg@uni-muenster.de \\ E-mail: ophileuni-muenster.de
}

Euclidean strong coupling expansion of the partition function is applied to lattice Yang-Mills theory at finite temperature, i.e. for lattices with a compactified temporal direction. The expansions have a finite radius of convergence and thus are valid only for $\beta<\beta_{c}$, where $\beta_{c}$ denotes the nearest singularity of the free energy on the real axis. The accessible temperature range is thus the confined regime up to the deconfinement transition. We have calculated the first few orders of these expansions of the free energy density as well as the screening masses for the gauge groups $\mathrm{SU}(2)$ and SU(3). The resulting free energy series can be summed up and corresponds to a glueball gas of the lowest mass glueballs up to the calculated order. Our result can be used to fix the lower integration constant for Monte Carlo calculations of the thermodynamic pressure via the integral method, and shows from first principles that in the confined phase this constant is indeed exponentially small. Similarly, our results also explain the weak temperature dependence of glueball screening masses below $T_{c}$, as observed in Monte Carlo simulations. Possibilities and difficulties in extracting $\beta_{c}$ from the series are discussed.

The XXV International Symposium on Lattice Field Theory

July 30-4 August 2007

Regensburg, Germany

* Speaker. 


\section{Introduction}

Contrary to weak coupling expansions, strong coupling expansions are known to be convergent series with a finite radius of convergence. In the early days of lattice gauge theory they were used to get analytical results for some physical quantities of interest, such as glueball masses or the energy density of lattice Yang-Mills theories. These calculations were done at zero temperature, i.e. at infinite volume $N_{s}^{3}$ and temporal extent $N_{t}$ of the lattice.

Here we calculate such series expansions for the free energy density and screening masses with an infinite spatial volume and a compactified temporal extension $N_{t}$ of the lattice. In this way finite temperature effects are generated, giving us the opportunity to study the physical, temperature dependent free energy density in the confined phase. The physical deconfinement phase transition then corresponds to a finite convergence radius of the series, which one may try to estimate from the behaviour of the coefficients.

\section{Free energy density}

\subsection{Cluster expansion}

The partition function of the lattice Yang-Mills theory is given by a functional integration of the exponentiated Wilson action over the corresponding $\mathrm{SU}(\mathrm{N})$ group space,

$$
\begin{aligned}
& Z=\int D U \exp \left[\sum_{p} \frac{\beta}{2 N}\left(\operatorname{Tr} U+\operatorname{Tr} U^{\dagger}-2 N\right)\right], \\
& \beta=\frac{2 N}{g^{2}} .
\end{aligned}
$$

An expansion in the lattice coupling $\beta$ by group characters $\chi_{r}(U)$ and a cluster expansion yields the free energy density [1]

$$
\tilde{f} \equiv-\frac{1}{\Omega} \ln Z=-6 \ln c_{0}(\beta)-\frac{1}{\Omega} \sum_{C=\left(X_{i}^{n_{i}}\right)} a(C) \prod_{i} \Phi\left(X_{i}\right)^{n_{i}} .
$$

where $\Omega=V \cdot N_{t}$ is the lattice volume and $c_{0}$ is the expansion coefficient of the trivial representation, which has been factored out. The combinatorial factor $a(C)$ is introduced via a momentcumulant-formalism, and equals 1 for clusters $C$ which consist of only one so-called polymer $X_{i}$. The quantity in eq. 2.2 is customarily called a free energy, even at zero physical temperature, because the path integral corresponds to a partition function if one formally identifies $\beta$ with $1 / T$. Here we are interested in a physical temperature $T=1 /\left(a N_{t}\right)$, realized by compactifying the temporal extension of the lattice. The physical free energy is then obtained by subtracting the formal $\left(N_{t}=\infty\right)$ free energy, which is analogous to a subtraction of the divergent vacuum energy in the continuum. Thus the physical free energy density reads

$$
f\left(N_{t}, u\right)=\tilde{f}\left(N_{t}, u\right)-\tilde{f}(\infty, u) .
$$

The contributing polymers $X_{i}$ have to be objects with a closed surface, since

$$
\int d U \chi_{r}(U)=\delta_{r, 0} .
$$




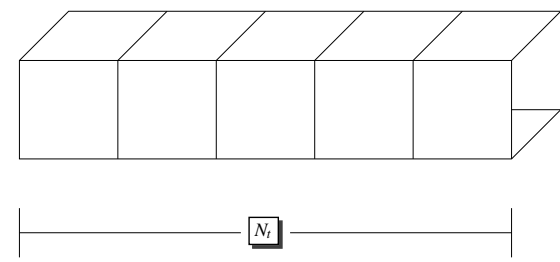

Figure 1: Graph contributing to the lowest order $f_{1}\left(N_{t}, u\right)$ of the expansion of the physical free energy density at finite temperature.

This means the group integration projects out the trivial representation at each link. To calculate the group integrals one uses the integration formula

$$
\int d U \chi_{r}(U V) \chi_{r}\left(W U^{-1}\right)=\chi_{r}(V W) .
$$

For a more detailed introduction to strong coupling calculations we refer to [1].

\subsection{Results}

The graph contributing to the lowest order of the free energy density is a tube of length $N_{t}$ with a cross-section of one single plaquette (fig. 1). The contribution of these tubes together with inner plaquettes is

$$
\begin{array}{ll}
\mathrm{SU}(2): & f_{1}\left(N_{t}, u\right)=-\frac{3}{N_{t}} u^{4 N_{t}} a^{N_{t}}, \\
\mathrm{SU}(3): & f_{1}\left(N_{t}, u\right)=-\frac{3}{N_{t}} u^{4 N_{t}}\left[b^{N_{t}}+c^{N_{t}}\right],
\end{array}
$$

where $u, v$ and $w$ are the expansion parameters of the lowest dimensional representations of the corresponding gauge groups,

$$
\begin{aligned}
& \mathrm{SU}(2): \quad u=\frac{\beta}{4}+\mathscr{O}\left(\beta^{2}\right) \quad v=\frac{\beta^{2}}{24}+\mathscr{O}\left(\beta^{4}\right), \\
& \mathrm{SU}(3): \quad u=\frac{\beta}{18}+\mathscr{O}\left(\beta^{2}\right) \quad v=\frac{\beta^{2}}{432}+\mathscr{O}\left(\beta^{4}\right) \quad w=\frac{\beta^{2}}{288}+\mathscr{O}\left(\beta^{4}\right),
\end{aligned}
$$

and we have used the abbreviations

$$
\begin{aligned}
& a=1+3 v-4 u^{2}, \\
& b=1-3 u-6 v+8 w \\
& c=1+3 u+6 v+8 w-18 u^{2} .
\end{aligned}
$$

Higher order contributions consist of such tubes with local decorations of additional plaquettes either in the fundamental or in higher representations. For the interesting cases $\mathrm{SU}(2)$ and $\mathrm{SU}(3)$, these contributions up to the calculated orders are

$$
\mathrm{SU}(2): \quad f\left(N_{t}, u\right)=-\frac{3}{N_{t}} u^{4 N_{t}} a^{N_{t}}\left[1+12 N_{t} u^{4}-\frac{1556}{81} N_{t} u^{6}+\left(86 N_{t}^{2}+\frac{35828}{243} N_{t}\right) u^{8}\right]
$$




$$
\begin{aligned}
\mathrm{SU}(3): \quad f\left(N_{t}, u\right)= & -\frac{3}{N_{t}} u^{4 N_{t}} c^{N_{t}}\left[1+12 N_{t} u^{4}+42 N_{t} u^{5}-\frac{115343}{2048} N_{t} u^{6}-\frac{1095327}{2048} N_{t} u^{7}\right] \\
& -\frac{3}{N_{t}} u^{4 N_{t}} b^{N_{t}}\left[1+12 N_{t} u^{4}+30 N_{t} u^{5}-\frac{17191}{256} N_{t} u^{6}+63 N_{t} u^{7}\right],
\end{aligned}
$$

which are valid only for $N_{t} \geq 5$. For smaller $N_{t}$ there are modifications of these formulae coming from polymers with cross-sections larger than one plaquette. The complete results for $N_{t}=2$ and 3 in $\mathrm{SU}(2)$ are

$$
\begin{aligned}
& N_{t}=2: \quad f(2, u)=-\frac{3}{2} u^{8}\left[1-4 u^{2}+\frac{110}{3} u^{4}-\frac{58472}{405} u^{6}+\frac{61529701}{65610} u^{8}\right], \\
& N_{t}=3: \quad f(3, u)=-u^{12}\left[1-6 u^{2}+50 u^{4}-\frac{37966}{135} u^{6}+\frac{843898}{405} u^{8}\right]
\end{aligned}
$$

\subsection{Free energy density as a glueball gas}

Recognizing the first orders of the corresponding glueball masses (see [2] and [3]) for SU(2)

$$
\begin{aligned}
& m\left(A_{1}^{++}\right)=-4 \ln u+2 u^{2}-\frac{98}{3} u^{4}-\frac{20984}{405} u^{6}-\frac{151496}{243} u^{8}, \\
& m\left(E^{++}\right)=-4 \ln u+2 u^{2}-\frac{26}{3} u^{4}+\frac{13036}{405} u^{6}-\frac{28052}{243} u^{8}
\end{aligned}
$$

and SU(3)

$$
\begin{aligned}
& m\left(A_{1}^{++}\right)=-4 \ln u-3 u+9 u^{2}-\frac{27}{2} u^{3}-7 u^{4}-\frac{297}{2} u^{5}+\frac{858827}{10240} u^{6}+\frac{47641149}{71680} u^{7}, \\
& m\left(E^{++}\right)=-4 \ln u-3 u+9 u^{2}-\frac{27}{2} u^{3}+17 u^{4}-\frac{153}{2} u^{5}+\frac{1104587}{10240} u^{6}+\frac{29577789}{71680} u^{7} \\
& m\left(T_{1}^{+-}\right)=-4 \ln u+3 u+\frac{9}{2} u^{3}-\frac{98}{4} u^{4}+\frac{33}{4} u^{5}-\frac{36771}{1280} u^{6}+\frac{117897}{448} u^{7}
\end{aligned}
$$

one can write

$$
\begin{aligned}
S U(2): & f\left(N_{t}, u\right) & =-\frac{1}{N_{t}}\left[e^{-m\left(A_{1}^{++}\right) N_{t}}+2 e^{-m\left(E^{++}\right) N_{t}}+\mathscr{O}\left(u^{4}\right)\right], \\
S U(3): & & f\left(N_{t}, u\right)=-\frac{1}{N_{t}}\left[e^{-m\left(A_{1}^{++}\right) N_{t}}+2 e^{-m\left(E^{++}\right) N_{t}}+3 e^{-m\left(T_{1}^{+-}\right) N_{t}}+\mathscr{O}\left(u^{4}\right)\right],
\end{aligned}
$$

corresponding to a gas of non-interacting glueballs in a hadron-resonance-gas model [4], where

$$
f \simeq-T \sum_{i} e^{-\frac{E_{i}}{T}}
$$

This is a rather remarkable result. It allows to see from first principles that the pressure $p=-f$ is exponentially small in the confined phase, and it explains the success of the hadron-resonancegas model in reproducing the confined phase equation of state. Since the partition function is not directly measurable in Monte-Carlo simulations, the pressure is usually obtained by the integral method [5], where the expectation values of derivatives are computed and then integrated numerically,

$$
\left.\frac{p}{T^{4}}\right|_{\beta_{0}} ^{\beta}=N_{t}^{4} \int_{\beta_{0}}^{\beta} d \beta^{\prime}\left[S_{0}-S_{T}\right],
$$



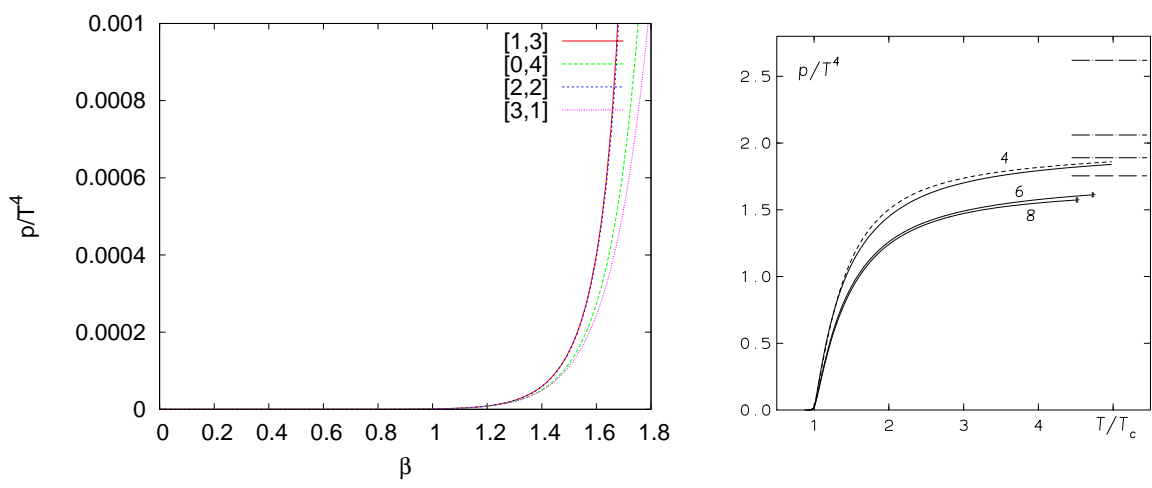

Figure 2: Left: Plot of the pressure density $p$ vs. $\beta\left(L+M=4\right.$ Padés for $\mathrm{SU}(2)$ and $N_{t}=3$. The plot range corresponds to the confined phase up to the critical coupling. Right: Plot of $p$ vs. $T / T_{c}$ for $\mathrm{SU}(3)$ from Monte Carlo data [5].

with $S_{0}=6 P_{0}$ and $S_{T}=3\left(P_{t}+P_{s}\right)$, where $P_{0}$ denotes the plaquette expectation value on symmetric lattices and $P_{t, s}$ are those of space-time and space-space plaquettes with $N_{t}<N_{s}$. The lower integration limit is usually set to zero by hand, arguing with an exponentially small pressure in the low temperature regime. Our results now justify this assumption from first principles.

\subsection{Phase transition}

Physical phase transitions limit the radius of convergence on the real $\beta$-axis, signalled by a singularity in the full free energy. We model the full function from the series coefficients by Padé approximants $[L, M]$ with

$$
[L, M](u) \equiv \frac{1+a_{1} u+\ldots+a_{L} u^{L}}{b_{0}+b_{1} u+\ldots+b_{M} u^{M}}
$$

and search for the zeroes of the denominator. The resulting $L+M=2,3,4$ Padé tables for $N_{t}=2,3$ with the nearest real singularities are shown in table 1.

Zeroes $u_{0}$ of the Padé approximant which are very close to a singularity often indicate that the singularity is superfluous and disappears as the full funtion is approached. Hence, removing the

$$
\text { SU(2): } N_{t}=2
$$

\begin{tabular}{|c|c|c|c|}
\hline$[L, M]$ & $u_{c}$ & $\beta_{c}$ & $\left|u_{c}-u_{0}\right|$ \\
\hline \hline$[1,2]$ & 0.4143 & 1.8865 & 0.0642 \\
{$[0,3]$} & 0.4675 & 2.2201 & \\
{$[2,2]$} & 0.5492 & 2.8350 & 0.3419 \\
{$[1,3]$} & 0.4753 & 2.2725 & 1.3038 \\
{$[0,4]$} & 0.4766 & 2.2816 & \\
\hline
\end{tabular}

$\mathbf{S U}(\mathbf{2}): N_{t}=3$

\begin{tabular}{|c|c|c|c|}
\hline$[L, M]$ & $u_{c}$ & $\beta_{c}$ & $\left|u_{c}-u_{0}\right|$ \\
\hline \hline$[1,2]$ & 0.3467 & 1.5133 & 0.0219 \\
{$[0,3]$} & 0.5009 & 2.4538 & \\
{$[2,2]$} & 0.4622 & 2.1853 & 0.2388 \\
{$[1,3]$} & 0.4347 & 2.0098 & 0.1373 \\
{$[0,4]$} & 0.4617 & 2.1820 & \\
\hline
\end{tabular}

Table 1: Zeroes of the denominator $\left(u_{c}\right)$ and the numerator $\left(u_{0}\right)$ of the $[L, M]$ Padé approximants and the corresponding value of $\beta_{c}$. 
singularities with a nearby zero, we obtain estimates for the critical couplings, which are not far from the Monte Carlo results $\beta_{c}=1.8800$ (30) for $N_{t}=2$ and $\beta_{c}=2.1768(30)$ for $N_{t}=3$ [6].

The $L+M=4$ Padé's for $N_{t}=3$, SU(2), are shown in figure (2). The spread in the curves gives an estimate of the systematic error of the approximants at that order. The exponential suppression in the confined phase as well as the onset of the pressure upon approaching $T_{c}$ is reproduced by the strong coupling series.

\section{Screening masses}

\subsection{Zero temperature}

Screening masses are defined by the exponential decay of the spatial correlation of suitable operators. We used plaquette operators in our calculations. Temporarily assigning separate gauge couplings to all plaquettes, the correlator can be defined as [2]

$$
C(z)=\left\langle\operatorname{Tr} U_{p_{1}}(0) \operatorname{Tr} U_{p_{2}}(z)\right\rangle=\left.N^{2} \frac{\partial^{2}}{\partial \beta_{1} \partial \beta_{2}} \ln Z\left(\beta, \beta_{1} \beta_{2}\right)\right|_{\beta_{1,2}=\beta} .
$$

At zero temperature the exponential decay is the same as for correlations in the time direction, and thus determined by the gluball masses, the lowest of which may be extracted as

$$
m=-\lim _{z \rightarrow \infty} \frac{1}{z} \ln C(z)
$$

The leading order graphs for the strong coupling series are shown in fig. 3. This leads to the lowest order contribution:

$$
C(z)=A u^{4 z}=A \mathrm{e}^{-m_{s} z} .
$$

Thus the leading order for the screening mass is given by

$$
m_{s}=-4 \ln u(\beta)
$$

\subsection{Finite temperature}

The graph contributing to the lowest order of the difference between the screening masses at zero and finite temperature is shown in figure (3). To lowest order the mass difference is

$$
\begin{aligned}
\Delta m_{s}\left(N_{t}\right) & =m_{s}\left(N_{t}\right)-m_{s}(\infty) \\
& =-\frac{2}{3} N_{t} u^{4 N_{t}-6}
\end{aligned}
$$

Thus one can see that the finite temperature effect on the screening mass is very small below $T_{c}$, as is also observed in Monte Carlo simulations (for references, see [7]). 

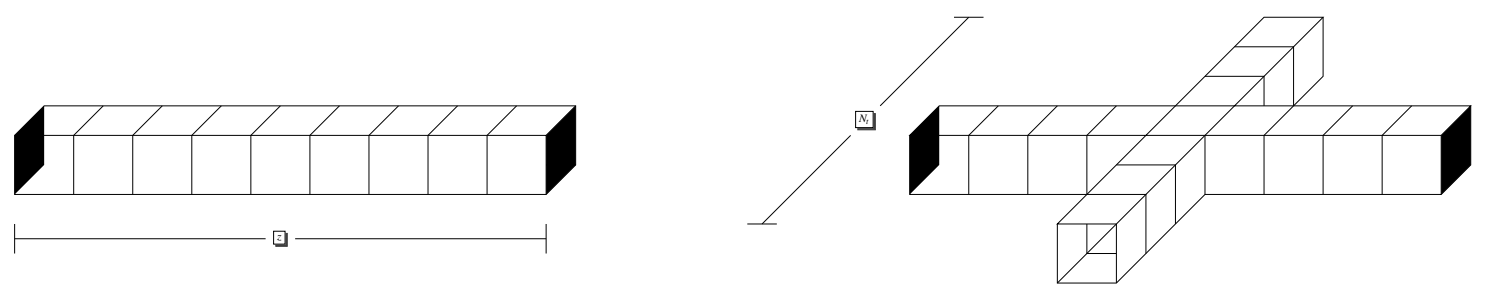

Figure 3: Graphs contributing to the lowest order of the expansion of the screening mass at vanishing and finite temperature. The correlation-plaquettes are painted black.

\section{Conclusions}

We performed explorative studies of strong coupling expansions at finite temperature. Our series for the free energy density is to the lowest orders consistent with a free glueball gas. This result justifies the neglect of the lower integration constant in numerical calculations of the equation of state by the integral method from first principles. Moreover, it gives an explanation for the success of the hadron-resonance-gas model in reproducing lattice data in the confined phase. By extrapolating the power series via Padé approximants and looking for the zeroes of the denominator, it is possible to get estimates for the critical value $\beta_{c}$ of the deconfining phase transition, although higher order terms seem necessary in order to obtain some accuracy here. Finally, glueball screening masses show a weak temperature dependence in the confined phase, consistent with what is found in numerical simulations.

\section{References}

[1] I. Montvay and G. Münster, Quantum fields on a lattice, Cambridge University Press, UK (1994).

[2] G. Münster, Strong Coupling Expansions For The Mass Gap In Lattice Gauge Theories, Nucl. Phys. B 190 (1981) 439.

[3] K. Seo, Glueball Mass Estimate By Strong Coupling Expansion In Lattice Gauge Theories, Nucl. Phys. B 209 (1982) 200.

[4] F. Karsch, K. Redlich and A. Tawfik, Thermodynamics at non-zero baryon number density: A comparison of lattice and hadron resonance gas model calculations, Phys. Lett. B 571 (2003) 67 [arXiv:hep-ph/0306208].

[5] G. Boyd, J. Engels, F. Karsch, E. Laermann, C. Legeland, M. Lütgemeier and B. Petersson, Thermodynamics of SU(3) Lattice Gauge Theory, Nucl. Phys. B 469 (1996) 419 [arXiv:hep-lat/9602007].

[6] J. Fingberg, F. Karsch and U. M. Heller, Scaling and asymptotic scaling in the SU(2) gauge theory, Nucl. Phys. Proc. Suppl. 30 (1993) 343 [arXiv: hep-lat/9208012].

[7] E. Laermann and O. Philipsen, Status of lattice QCD at finite temperature, Ann. Rev. Nucl. Part. Sci. 53 (2003) 163 [arXiv: hep-ph/0303042]. 